\title{
High Quality ZnO Films Deposited by PLD Technique with Bias Voltages*
}

\author{
Takao Komiyama ${ }^{\dagger}$ \\ Department of Electronics and Information Systems, Akita Prefectureal University, \\ 84-4 Tsuchiya-Ebinoguch,i Yuri-Honjo, Akita 015-0055, Japan and \\ Graduate School of Engineering, Iwate University, 4-3-5, Ueda, Morioka, Iwate 020-8551, Japan \\ Kouji Iwataki, Hiroyuki Yamaguchi, and Takashi Aoyama \\ Department of Electronics and Information Systems, Akita Prefectureal University, \\ 84-4 Tsuchiya-Ebinoguchi, Yuri-Honjo, Akita 015-0055, Japan $\ddagger$ \\ Yasube Kashiwaba \\ Center for Regional Collaboration in Research and Education, \\ Iwate University, 4-3-5, Ueda, Morioka, Iwate 020-8551, Japan \\ Hiroshi Taniguchi \\ Department of Welfare Engineering, Iwate University, 4-3-5, Ueda, Morioka, Iwate 020-8551, Japan
}

(Received 5 June 2008; Accepted 8 October 2008; Published 4 April 2009)

\begin{abstract}
The effects of the PLD bias voltage technique on the $\mathrm{ZnO}$ film quality have been investigated. The ZnO films were grown on sapphire substrates under bias voltages and oxygen atmosphere conditions. A molybdenum mesh electrode is set between the substrate and the target. The bias voltages and the oxygen pressures were changed between $\pm 500 \mathrm{~V}$ and between $10^{-2}$ and $10^{-6}$ Torr, respectively. Photoluminescence measurement showed that the defect density had minimum values when the bias voltage was about $+200 \mathrm{~V}$ and the oxygen pressure was $10^{-2}$ Torr. The optimum bias voltages decrease from +400 to $+200 \mathrm{~V}$ by using oxygen atmospheres. Because the defect densities are decreased significantly by applying bias voltages under the optimized oxygen condition, the reasons of the improved $\mathrm{ZnO}$ film quality comes from not only the compensation of the decrease in the oxygen ratio in the film but also the decrease of the film damages caused by the high energy particles in the plume.
\end{abstract}

[DOI: $10.1380 /$ ejssnt.2009.294]

Keywords: Zinc oxide; Photoluminescence; LITD; Semiconducting films; Electrical transport

\section{INTRODUCTION}

$\mathrm{ZnO}$ is one of the most promising materials for optoelectronic devices such as ultraviolet (UV) light emitting diodes (LEDs) and laser diodes (LDs) [1]. As $\mathrm{ZnO}$ is a non-stoichiometoric material due to a number of defects, it is still difficult to attain stable $p$-type conduction. Therefore, it is urgent to decrease significantly the defect density of the $\mathrm{ZnO}$ films. Up to date, a pulsed laser deposition (PLD) technique is one of the simple methods to obtain middle quality films. However, when $\mathrm{ZnO}$ films are grown by a conventional PLD technique, the oxygen ratio in the films is decreased, and to compensate this, the PLD chamber is usually kept in the oxygen atmosphere of about $10^{-2}$ Torr. Even when the $\mathrm{ZnO}$ films are grown in the oxygen atmospheres, the film quality is still not enough to attain stable $p$-type conduction.

About the improvement of sputtering techniques, the methods of applying bias voltages to the substrates have been widely investigated [2-4]. Doh et al. reported that applying bias voltages was surely effective to improve the

\footnotetext{
*This paper was presented at the 14th International Conference on Solid Films and Surfaces (ICSFS-14), Trinity College Dublin, Ireland, 29 June - 4 July, 2008.

$\dagger$ Corresponding author: komiyama@akita-pu.ac.jp

${ }^{\ddagger}$ Present address: School of materials Science, Japan Advanced Institute of Science and Technology, 1-1 Asahidai, Nomi, Ishikawa 923-1292, Japan
}

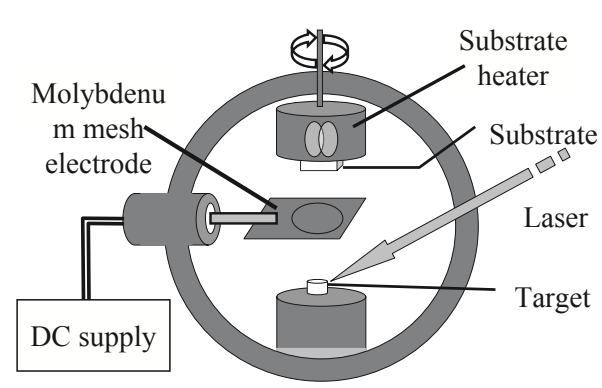

FIG. 1: Schematic illustration of PLD technique with bias voltage.

$\mathrm{ZnO}$ film quality [5]. About the improvement of the PLD technique, we have already reported that this bias voltage technique was also effective to decrease the defect density and to increase the near band edge (NBE) emission of the $\mathrm{ZnO}$ films [6]. However, the PLD technique is different from the conventional sputtering technique in that the PLD utilize the high energy laser excitation, and the mechanism for the improved film quality is not clarified yet.

In this investigation, we have grown the PLD $\mathrm{ZnO}$ films under both bias voltages and oxygen atmosphere conditions, and we found that the effects of the bias voltages are not only to optimize the oxygen ratio of the films but also to decrease the film damages caused by high energy particles in the PLD plume. 


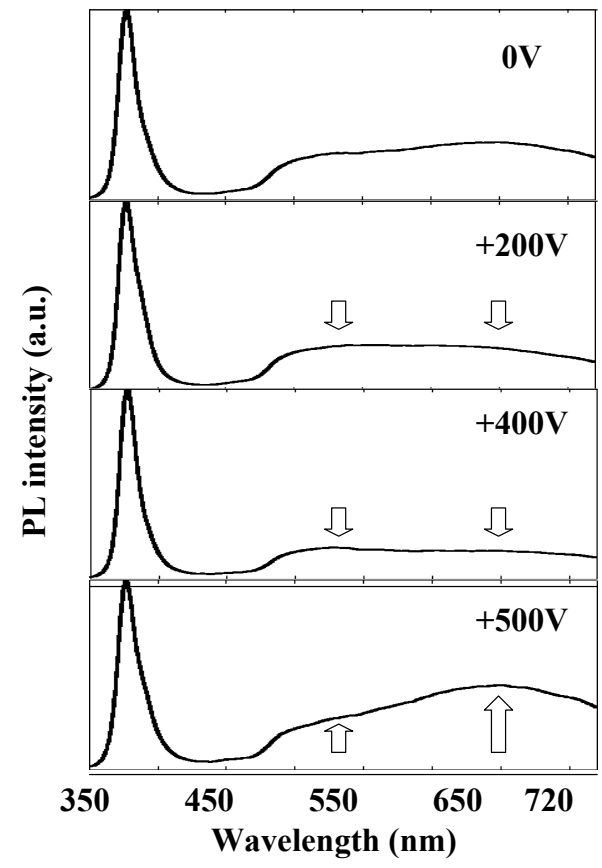

FIG. 2: The PL spectra of the $\mathrm{ZnO}$ films grown under positive bias voltages and under oxygen-free conditions.

\section{EXPERIMENTAL}

Figure 1 shows a schematic illustration of PLD technique with bias voltages. A molybdenum mesh electrode is set between the substrate and the target. The molybdenum mesh is made of a molybdenum plate with holes of $15 \mathrm{~mm}$ in diameter. The molybdenum plate size is $5 \mathrm{~mm}$ square and the molybdenum wire is $0.1 \mathrm{~mm}$ in diameter. The $\mathrm{ZnO}$ (99.99\%) targets were purchased from Kojundo Chemical Laboratory Co. Ltd. The target size is $10 \mathrm{~mm}$ in diameter and $5 \mathrm{~mm}$ in thickness. The molybdenum mesh electrode was placed $27 \mathrm{~mm}$ above the target. $\mathrm{ZnO}$ films were grown on sapphire substrates $(10 \mathrm{~mm} \times 10 \mathrm{~mm})$. The substrate was set $18 \mathrm{~mm}$ above the electrode and the substrate temperature was kept at $700^{\circ} \mathrm{C}$. The DC bias voltages between $-500 \mathrm{~V}$ and $+500 \mathrm{~V}$ were applied between the molybdenum electrode and the target. The oxygen pressure was changed between $10^{-2}$ Torr and $10^{-5}$ Torr. A Nd:YAG laser $(266 \mathrm{~nm})$ was used for the ablation; the pulse duration of $10 \mathrm{~ns}$, the repetition rate of 10 $\mathrm{Hz}$ and the output energy of $75 \mathrm{~mJ}$. The base pressure of the chamber is $10^{-7}$ Torr. The x-ray diffraction (XRD) measurement was done in the Brngg-Brentano model using $\mathrm{Cu}$ Ka radiation. Photoluminescence (PL) spectra were measured in air at room temperatures using FICS type spectrometer (Oriel, Ltd.) and Newton type CCD detector (Andor, Ltd.). An excitation source of PL measurement was $325 \mathrm{~nm}$ He-Cd laser with an output power of $30 \mathrm{~mW}$.

\section{RESULTS AND DISCUSSION}

Figure 2 shows the PL spectra of the films grown under positive biases and oxygen-free $\left(10^{-5}\right.$ Torr $)$ conditions.

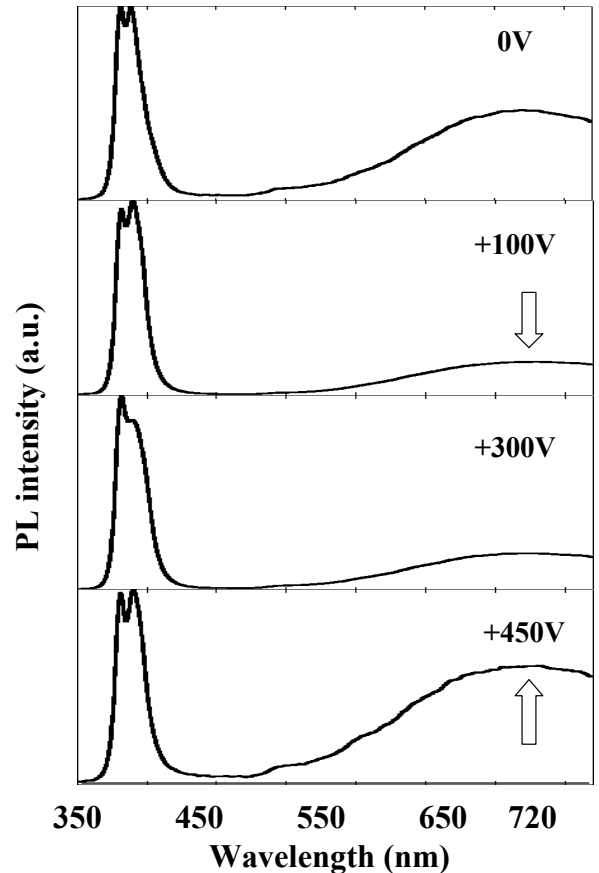

FIG. 3: The PL spectra of $\mathrm{ZnO}$ films grown under ositivebias voltages and oxygen atmosphere $\left(10^{-2}\right.$ Torr $)$ conditions.

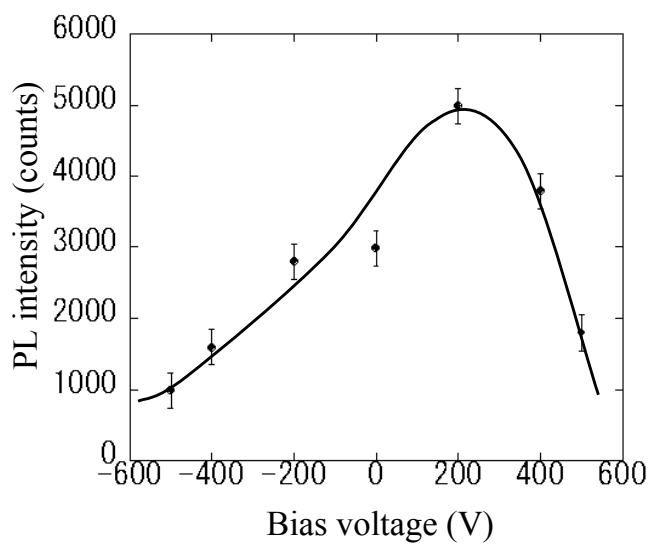

FIG. 4: The PL intensities of the near band edge (NBE) emission from $\mathrm{ZnO}$ films grown under oxygen-free $\left(10^{-5}\right.$ Torr $)$ conditions as a function of the bias voltages.

Three main peaks are observed at around $360 \mathrm{~nm}$ (the near band edge), $550 \mathrm{~nm}$ (the green band) and $670 \mathrm{~nm}$ (the red band). The peak intensities are normalized for the intensity at $360 \mathrm{~nm}$ (at the near band edge). As the bias voltage increases from 0 to $+400 \mathrm{~V}$, both the green and the red peaks are decreased. For the $500 \mathrm{~V}$ bias, the two peaks are increased. Figure 3 shows the PL spectra of the films grown under positive biases and oxygen atmosphere $\left(10^{-2}\right.$ Torr $)$ conditions. The intensity of the green band emission is significantly reduced for +100 and +200 $\mathrm{V}$ biases. The origin of the green band emission is suggested to come from either oxygen-vacancy or interstitial zinc related defects. The intensity of the red band emission is decreased for low $(+100$ and $+200 \mathrm{~V})$ bias voltages and it is increased for the high $(+450 \mathrm{~V})$ bias voltage as in Fig. 2. Figures 4 and 5 show the absolute intensities of 


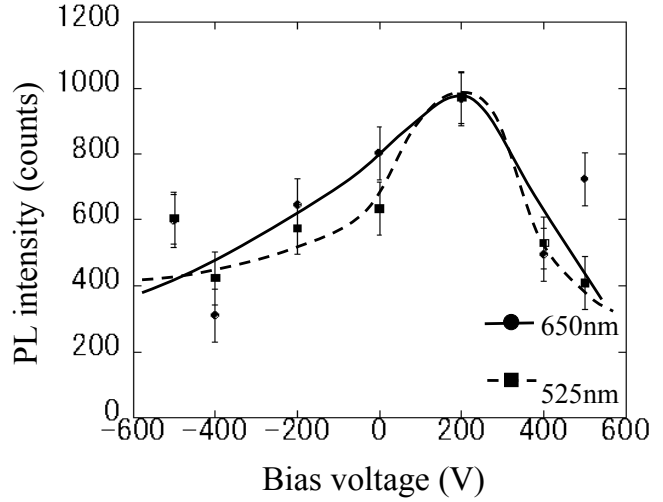

FIG. 5: The PL intensities of the defect (the green and the red) emission from $\mathrm{ZnO}$ films grown under oxygen-free $\left(10^{-5}\right.$ Torr $)$ conditions as a function of the bias voltages.

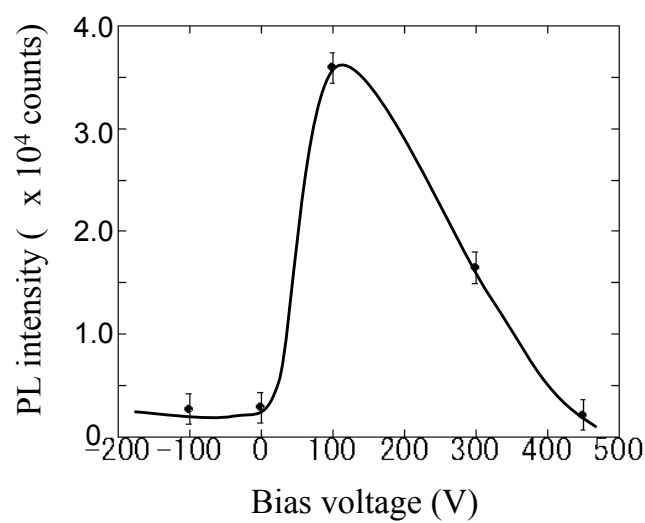

FIG. 6: The PL intensities of the near band edge (NBE) emission from $\mathrm{ZnO}$ films grown under the oxygen atmosphere $\left(10^{-2}\right.$ Torr $)$ condition as a function of the bias voltages.

the near band edge and the defect related emission under the oxygen-free condition. Both the intensities have maximum values for the $+200 \mathrm{~V}$ bias voltage. Figures 6 and 7 show the absolute intensities of the near band edge and the defect related emission under the oxygen atmosphere condition. Both the intensities have maximum values for the $+100 \mathrm{~V}$ bias voltage. The optimum bias voltage seems to shift from +200 to $+100 \mathrm{~V}$ for the films grown under the oxygen atmosphere. Figures 8 and 9 show the normalized areas of the defect emission as a function of the bias voltages. The normalized areas of the defect emission are decreased significantly by applying bias voltages under both oxygen-free and the oxygen atmosphere conditions. The optimum bias voltage is $+400 \mathrm{~V}$ for the films grown under oxygen-free condition and it is decreased to about $+200 \mathrm{~V}$ for the films grown under the oxygen atmosphere. The optimum bias voltages are slightly different between the absolute intensities in Figs. 4 to 7 , and the normalized areas in Figs. 8 and 9, however, the oxygen atoms seem to decrease the necessary bias voltages.

From these results, the bas voltages in the PLD technique seems to attract the ionized anions (ionized oxygen atoms, $\mathrm{O}^{2-}$ ) and repel the cations (ionized zinc atoms, $\mathrm{Zn}^{2+}$ ), and increase the oxygen ratio of the $\mathrm{ZnO}$ films. The oxygen atmosphere also increases the oxygen ratio of

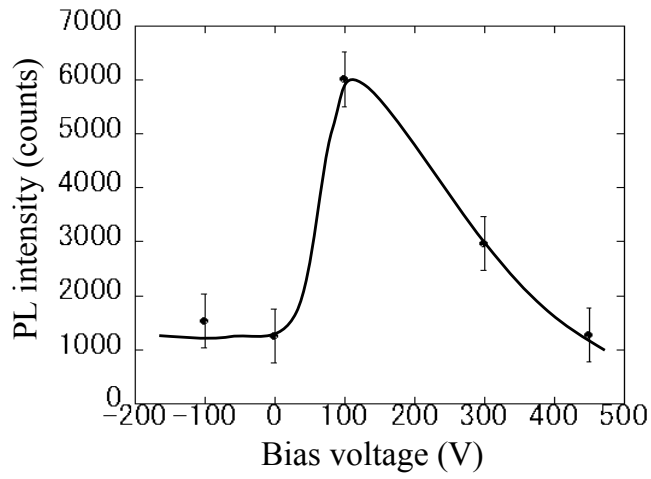

FIG. 7: The PL intensities of the defect (the green and the red) emission from $\mathrm{ZnO}$ films grown under the oxygen atmosphere $\left(10^{-2}\right.$ Torr $)$ condition as a function of the bias voltages.

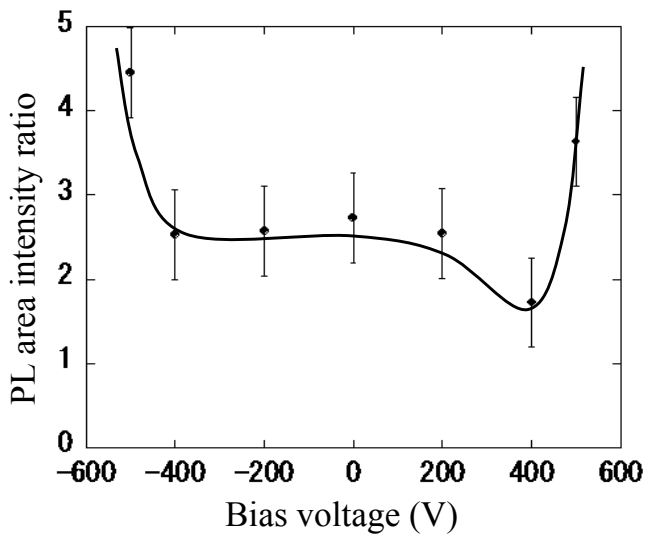

FIG. 8: The normalized areas of the PL from the defect (the green and the red) under the oxygen-free $\left(10^{-5}\right.$ Torr $)$ conditions as a function of the bias voltages.

the $\mathrm{ZnO}$ films. In our experiment, the oxygen atmosphere is optimized at $10^{-2}$ Torr of oxygen partial pressure. Figures 8 and 9 indicate that the bias voltages increase the near band emission intensities and that they decrease the normalized defect areas. The mechanism for the improved film quality cannot be explained simply by the optimized oxygen ratio in the $\mathrm{ZnO}$ films. As the atomic weight of zinc is about 4 times larger than that of oxygen, the high energy zinc ions $\left(\mathrm{Zn}^{2+}\right)$ in the plume are suggested to give a lot of damages to the $\mathrm{Zn}-\mathrm{O}$ bonds in the film. When the bias voltages are applied in the PLD chamber, the high energy zinc ions $\left(\mathrm{Zn}^{2+}\right)$ are repelled and the oxygen ions $\left(\mathrm{O}^{2-}\right)$ are attracted. The high energy bombardment by $\mathrm{Zn}^{2+}$ is reduced and the oxygen ratio in the film is increased. The PLD bias voltage technique seems to have two effects; one is to compensate the decrease in the oxygen ratio in the $\mathrm{ZnO}$ film and the other is to decrease the film damages caused by high energy particles in the plume.

\section{CONCLUSION}

The effects of the PLD bias voltage technique on the $\mathrm{ZnO}$ film quality have been investigated. The $\mathrm{ZnO}$ films 


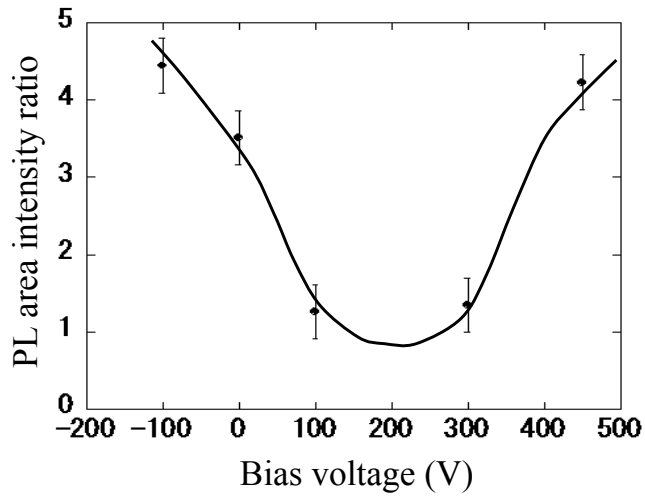

FIG. 9: The normalized areas of the PL from the defect (the green and the red) under the oxygen atmosphere $\left(10^{-2}\right.$ Torr $)$ condition as a function of the bias voltages. were grown on sapphire substrates under bias voltages and oxygen atmosphere conditions. A molybdenum mesh electrode is set between the substrate and the target. The bias voltages and the oxygen pressures were changed between $\pm 500 \mathrm{~V}$ and between $10^{-2}$ and $10^{-6}$ Torr, respectively. Photoluminescence measurement showed that the defect density had minimum values when the bias voltage was about $+200 \mathrm{~V}$ and the oxygen pressure was $10^{-2}$ Torr. The optimum bias voltages decrease from +400 to +200 $\mathrm{V}$ by using oxygen atmospheres. Because the defect densities are decreased significantly by applying bias voltages under the optimized oxygen condition, the reason for the improved $\mathrm{ZnO}$ film quality comes from not only the compensation of the decrease in the oxygen ratio in the film but also the decrease of the film damages caused by the high energy particles in the plume.
[1] M. Kawasaki, A. Ohtomo, I. Ohkubo, H. Koinuma, Z. K. Tang, P. Yu, G. K. L. Wong, B. P. Zhang, and Y. Segawa, Mater. Sci. Eng. B 56, 239 (1998).

[2] G. A. Rozgonyi and W. J. Polito, J. Vac. Sci. Technol. 6 115 (1969).

[3] F. Claeyssens, A. Cheesmasn, S. J. Henley, and M. N. R. Ashford, J. Appl. Phys. 92, 6886 (2002).
[4] L. M. Kukreja, A. Rohlfing, P. Misra, F. Hillenkamp, and K. Dreisewerd, Appl. Phys. A 78, 641 (2004).

[5] S. J. Doh, S. I. Park, T. S. Cho, and J. H. Je, J. Vac. Sci. Technol. A 17, 3003 (1999).

[6] H. Yamaguchi, T. Komiyama, M. Yamada, K. Sato, and T. Aoyama, Physica B 401-402, 391 (2007). 